\title{
Reversal of the Magnetic Nanofilms and Data Recording by Spin Current
}

\author{
Mykola M. Krupa \\ Institute of Magnetism National Academy of Science of Ukraine, Kiev 03143, Ukraine
}

\begin{abstract}
The article presents the results of experimental studies of the physical mechanisms and magnetic switching dynamics of films with one or two magnetic nanolayers under an irradiation picosecond and femtosecond laser pulses and also the samples of data recording devices on the spin storage medium are described. The study used a film with perpendicular anisotropy $\left(\mathrm{Tb}_{22} \mathrm{Co}_{5} \mathrm{Fe}_{73} / \mathrm{Pr}_{6} \mathrm{O}_{11} / \mathrm{Tb}_{19} \mathrm{Co}_{5} \mathrm{Fe}_{76}, \mathrm{~Tb}_{25} \mathrm{Co}_{5} \mathrm{Fe}_{70} / \mathrm{Al}_{2} \mathrm{O}_{3}, \mathrm{~Tb}_{22} \mathrm{Co}_{5} \mathrm{Fe}_{73}, \mathrm{~Tb}_{19} \mathrm{Co}_{5} \mathrm{Fe}_{76}\right)$ and films planar single-axis magnetic anisotropy $\left(\mathrm{Co}_{80} \mathrm{Fe}_{20} / \mathrm{Pr}_{6} \mathrm{O}_{11} / \mathrm{Co}_{30} \mathrm{Fe}_{70}\right)$. The magnetic switching of magnetic layers under action the magnetic field of a spin current is the most important for practical use in elements of spintronic. The spin current can also be realized using short electrical pulses. On the basis of this mechanism, the high-speed recording of information on the spin carrier has been realized.
\end{abstract}

Key words: Magnetic nanofilms, laser pulses, spin current, storage medium.

\section{Introduction}

Spintronics is based on the transport processes of spin-polarized current between the elements of electronic devices. The main efforts of researchers in the field of spintronics are aimed at searching for magnetic materials with a high degree of spin polarization and the development of apparatuses of electron spin orientation and the spin current filtration. Magnetic semimetals, magnetic Haussler alloys and magnetic semiconductors have high degree of spin polarization $[1,2]$. However, most of the non-magnetic semiconductors have, and are very difficult to get the mina uniform magnetic state by doping magnetic impurities. Technology for producing semi-metallic materials, which include Heusler alloys, are also quite complex.

It is necessary to understand, that the devices of spintronics can replace similar devices of microelectronics in rather limited areas of technology.

Corresponding author: Mykola M. Krupa, doctor, research fields: spintronics, magnetic characteristics and spin transport in multilayered magnetic nanofilms, influence of laser radiation, the effect of laser radiation on the structure, optical, electrical and magnetic characteristics of materials, nonlinear optic and generation of terrahertz radiation. E-mail: krupa@imag.kiev.ua.
First of all, it is the area of registration and processing of small constant and high-frequency electromagnetic signals. Therefore, for creation of elements of spintronics, multilayer heterogeneous metallic ferromagnetic nanostructures are used now. The elements of spintronics based on such metallic nanostructures are compared to the semiconductor elements of microelectronics more low noise, highthermal stability factor and can be used in a higher frequency range. For creation of high-frequency elements of spintronics, high-speed systems for controlling by a spatially localized magnetization are necessary. Usually magnetic reversal of magnetic films is realized with the help of itself magnetic field of the electric current flowing through special electrodes. However, in such simple way, it is difficult to create a considerable high-frequency magnetic field in small micro- and nano area.

This article presents the results of studies of the mechanisms and magnetic switching dynamics of films with one or two the magnetic nanolayers underan irradiation picosecond and femtosecond laser pulses and also the samples of data recording devices on the spin storage medium are described. 


\section{Investigated Films and Measuring Technique}

We have studied magnetic switching dynamics of the magnetic nanolayers in films with one or two magnetic nanolayers with perpendicular anisotropy $\left(\mathrm{Tb}_{25} \mathrm{Co}_{5} \mathrm{Fe}_{70} / \mathrm{Al}_{2} \mathrm{O}_{3}, \quad \mathrm{~Tb}_{22} \mathrm{Co}_{5} \mathrm{Fe}_{73}, \quad \mathrm{~Tb}_{19} \mathrm{Co}_{5} \mathrm{Fe}_{76}\right.$, $\left.\mathrm{Tb}_{22} \mathrm{Co}_{5} \mathrm{Fe}_{73} / \mathrm{Pr}_{6} \mathrm{O}_{11} / \mathrm{Tb}_{19} \mathrm{Co}_{5} \mathrm{Fe}_{76}\right)$ and films with planar single-axis magnetic anisotropy $\left(\mathrm{Co}_{80} \mathrm{Fe}_{20} / \mathrm{Pr}_{6} \mathrm{O}_{11} / \mathrm{Co}_{30} \mathrm{Fe}_{70}\right)$. Uniaxial anisotropy in the plane of the film with a small angular dispersion $(\Delta \alpha \approx$ $3^{\circ}$ ) is produced by special sputtering technique. Thicknesses of magnetic layers $\mathrm{TbCoFe}$ and $\mathrm{CoFe}$ constituted $20 \mathrm{~nm}$. For the barrier layer $\operatorname{Pr}_{6} \mathrm{O}_{11}$ and the cover layer $\mathrm{Al}_{2} \mathrm{O}_{3}$ that, thickness constitutes 2-3 nm and $40 \mathrm{~nm}$, respectively. Besides, we studied the influence of the laser pulse on the conductivity of the tunnel microcontacts: $\mathrm{Tb}_{22} \mathrm{Co}_{5} \mathrm{Fe}_{73} / \mathrm{Pr}_{6} \mathrm{O}_{11} / \mathrm{Tb}_{19} \mathrm{Co}_{5} \mathrm{Fe}_{76}$ and $\mathrm{Co}_{80} \mathrm{Fe}_{20} / \mathrm{Pr}_{6} \mathrm{O}_{11} / \mathrm{Co}_{30} \mathrm{Fe}_{70}$. The tunnel contacts with a conductive surface $S \approx 20 \mu^{2}$ are produced by a photolithography. The difference of terbium and iron $\mathrm{TbCoFe}$ films and $\mathrm{Co}$ and $\mathrm{Fe}$ in the CoFe films allows obtaining films with different coercivity. The coercive force $H$ is equal at $T=300 \mathrm{~K}$ for $\mathrm{Tb}_{22} \mathrm{Co}_{5} \mathrm{Fe}_{73}$ films $H_{1}$ $\approx 3 \times 10^{5} \mathrm{~A} / \mathrm{m}$, for $\mathrm{Tb}_{19} \mathrm{Co}_{5} \mathrm{Fe}_{76} H_{2} \approx 1.2 \times 10^{5} \mathrm{~A} / \mathrm{m}$, for $\mathrm{Co}_{30} \mathrm{Fe}_{70} H_{1} \approx 25 \times 10^{2} \mathrm{~A} / \mathrm{m}$ and for $\mathrm{Co}_{80} \mathrm{Fe}_{20} H_{2} \approx 6$ $\times 10^{3} \mathrm{~A} / \mathrm{m}$. That allowed a magnetic switching of low coercive layers $\mathrm{Tb}_{19} \mathrm{Co}_{5} \mathrm{Fe}_{76}$ and $\mathrm{Co}_{30} \mathrm{Fe}_{70}$ by the external magnetic field without changing the magnetization direction of high coercive layers $\mathrm{Tb}_{22} \mathrm{Co}_{5} \mathrm{Fe}_{73}$ and $\mathrm{Co}_{80} \mathrm{Fe}_{20}$. Magnetic field direction coincided with an easy magnetization axis and its value varied from 0 to $8 \times 10^{5} \mathrm{~A} / \mathrm{m}$.

The high energy of the perpendicular anisotropy and considerable coercive force [3-5] ensure a long lifetime for the magnetization state in amorphous ferrimagnetic films $\mathrm{Tb}_{25} \mathrm{Co}_{5} \mathrm{Fe}_{70}, \quad \mathrm{~Tb}_{22} \mathrm{Co}_{5} \mathrm{Fe}_{73}$ and $\mathrm{Tb}_{19} \mathrm{Co}_{5} \mathrm{Fe}_{76}$, which is close to saturation magnetization even in the zero magnetic fields. This allows the use of such films in the magnetized state as the CoFe [6] as the spin current injectors. Magnetic properties of these films make them a good model for the study of the characteristics of magnetic switching and provide commendation for the technology of spintronics elements.

In our researches, the scheme of measurements of Faraday angle and Kerr angle $[5,7]$ and picosecond $\left(\tau_{I}\right.$ $\approx 80 \mathrm{ps}$ ) and femtosecond ( $\tau_{i} \approx 130 \mathrm{fs}$ ) laser pulses and pump-prob technique were used. We also study the influence of these laser pulses on resistance of microcontacts $\mathrm{Tb}_{19} \mathrm{Co}_{5} \mathrm{Fe}_{76} / \mathrm{Pr}_{6} \mathrm{O}_{11} / \mathrm{Tb}_{22} \mathrm{Co}_{5} \mathrm{Fe}_{73}$ and $\mathrm{Co}_{80} \mathrm{Fe}_{20} / \mathrm{Pr}_{6} \mathrm{O}_{11} / \mathrm{Co}_{30} \mathrm{Fe}_{70}$. The magnetic electrodes of microcontact magnetized by an external magnetic field in one direction or towards one another, and irradiated with laser pulses.

\section{Mechanism and Dynamic of Magnetization Reversal of Magnetic Films by Laser Radiation}

Irradiation of magnetic films laser pulses results change of their characteristics under the influence of thermal and nonthermal physical processes. In the case of the thermal action, laser heating of the nanolayer considerably reduces its coercive force that can lead to magnetic reversal of this layer under the influence of an external magnetic field or a demagnetizing field. In ferromagnetic films, it is observed high-speed thermal magnetic switching [8,9]. Such magnetic reversal without external magnetic field associated with the processes of thermal demagnetization sublattice ferrimagnetic material, which have the opposite magnetization orientation. Laser-induced thermal demagnetization of each magnetic sublattice is in their antiferromagnetic exchange interaction. Further magnetic reversal of a magnetic film occurs under the influence of an internal field of antiferromagnetic interaction between magnetic sublattices rare-earth and transitive metals. However, it should be noted that in ferromagnetic films with perpendicular anisotropy, the magnetization reversal process heat are important field demagnetization $H_{d m}$ emerging in the region of action of laser radiation. The demagnetizing field in the region of diameter $d_{0}$ and thickness $h$ of a magnetic 
nanolayer heated to the Curie temperature can be estimated using the expression $H_{d m} \approx H_{a} h / d_{0}$, where, $H_{a}$ is the coercive force of the magnetic nanolayer.

The major mechanism of nonthermal magnetic reversal of films, with perpendicular anisotropy is the inverse Faraday effect $[10,11]$. This mechanism is realized only at the circular polarization of laser radiation and the created magnetic field $H_{F}$ proportional to the intensity of light $I$. In the thin conducting magnetic films can be found field $H_{F}$ on the basis of a circular current induced by laser radiation [11].

$$
\begin{aligned}
H_{F} & =\frac{\left|\vec{M}_{F}\right| \mu}{4 \pi \mu_{0}}=\frac{e^{3} \mu N E^{2}}{16 \pi \mu_{0} n\left(m_{e}^{*}\right)^{2} \omega^{3}} \\
& =\frac{e^{3} \mu N I}{8 \pi \mu_{0} c \varepsilon_{0} n\left(m_{e}^{*}\right)^{2} \omega^{3}}
\end{aligned}
$$

where, $\omega$ is a laser frequency; $c$ is the light speed; $m_{e}{ }^{*}$ and $N$ are effective masse and concentration of conductive electrons; $\varepsilon$ and $\mu$ are dielectric and magnetic constant. By using laser radiation with the right circular polarization or the left circular polarization, we can obtain the magnetic field directed towards the laser beam or against it. The calculated value of $H_{F}$ can reach large values $H_{F}>10^{5}-10^{6} \mathrm{~A} / \mathrm{m}$ for films with $\mu=10^{3}-10^{4}$ and $N \approx 10^{22} \mathrm{~cm}^{-3}$ and $I=$ $10^{9} \mathrm{~W} / \mathrm{cm}^{2}$. The characteristic relaxation time for the inverse Faraday effect is equal to $10^{-13}-10^{-14} \mathrm{~s}$.

Magnetic reversal of the second and next magnetic layer can pass in multilayered magnetic nanostructures under the influence of the new physical mechanism, connected with injection in this layer the spin polarized electrons. These polarized electrons produces in the second magnetic layer, the nonequilibrium magnetic moment $M_{i}$ and appropriate nonequilibrium magnetic field $H_{i}[12,13]$. This field consists of the self-field $H_{e}$ of the electric current and magnetic field $H_{s}$ related to the total magnetic moment of the injection spins $H_{i}=H_{e}+H_{s}$. These magnetic fields have different directions: the field $H_{e}$ lies in the plane of the film, while the field $H_{s}$ is directed to the magnetization $\mathrm{M}_{1}$ of the first magnetic layer. At perpendicular anisotropy of the first magnetic layer $H_{S}$ is directed normally to the second magnetic layer. The results of the article [12] showed that the relation $H_{e} / H_{s} \sim d_{0}\left(d_{0}\right.$ is the diameter of the current conductor). The laser-induced injection of spin polarized electrons

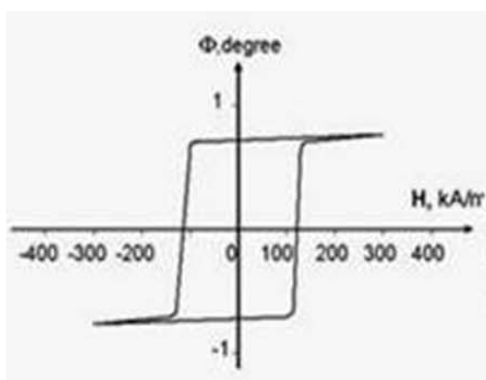

(a)

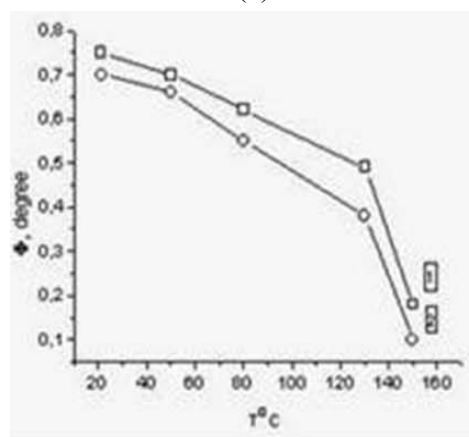

(d)

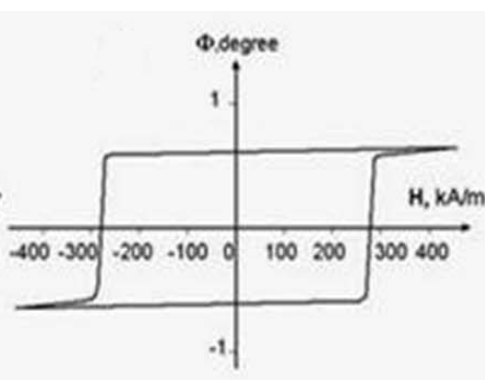

(b)

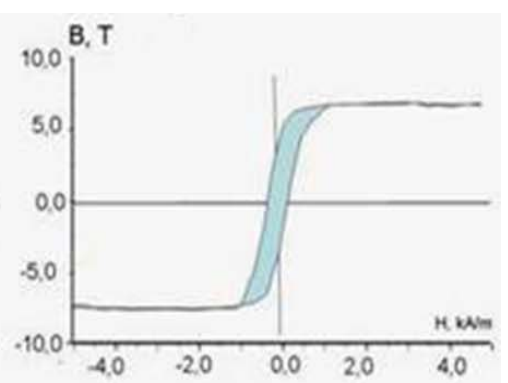

(c)

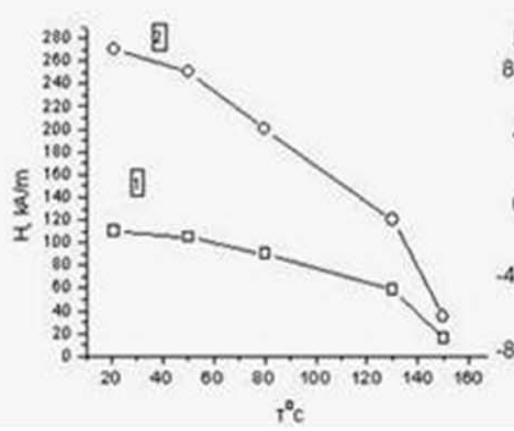

(e)

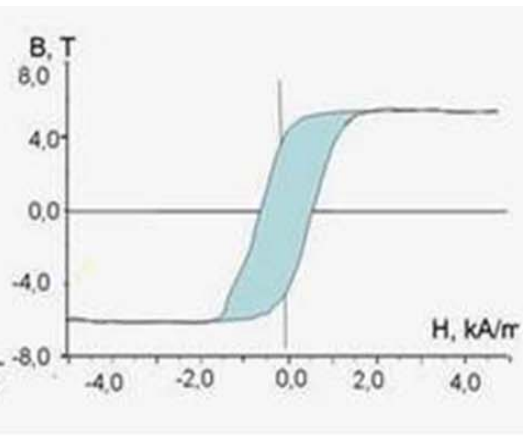

(f)

Fig. 1 The curves of change of the Kerr angle $(\Phi)$ in nanolayers $\mathrm{Tb}_{19} \mathrm{Co}_{5} \mathrm{Fe}_{76}(\mathrm{a}, \mathrm{e}-1), \mathrm{Tb}_{22} \mathrm{Co}_{5} \mathrm{Fe}_{73}(\mathrm{c}, \mathrm{e}-2)$ and $\mathrm{Tb}_{25} \mathrm{Co}_{5} \mathrm{Fe}_{70}$ and the B-H curves of nanolayers $\mathrm{Co}_{80} \mathrm{Fe}_{20}(\mathrm{c})$ and $\mathrm{Co}_{30} \mathrm{Fe}_{70}(\mathrm{f})$. 
is raised in a multilayered magnetic nanofilm under the influence of the photon drag effect $[5,14,15]$. Since the laser pulse duration $\tau_{i}$ is larger or comparable with the pulse $\tau_{p}$ and the spin relaxation time $\tau_{s}$ in the magnetic layer, the magnetic field $\mathrm{Hs}$ and $\mathrm{He}$, can be described by the estimating expression [5]:

$$
\begin{aligned}
& H_{s}=l_{s} \alpha(1-R) I \mu_{B} \mu \frac{n_{0} \tau_{p} \tau_{s} \gamma \eta \xi}{2 \mu_{0} h_{2} m_{e} c}, \\
& H_{e}=l_{e} \alpha(1-R) \operatorname{Ier} \frac{n_{0} \tau_{p} \gamma \xi}{4 \pi m_{e} c}
\end{aligned}
$$

where, $\alpha, R$ and $n_{0}$ are absorption and reflection coefficients and refractive index respectively; $h_{1}$ and $m_{\mathrm{e}}$ are the thickness of the first magnetic layer and effective electron mass; $\gamma<1, \eta<1$ and $\xi<1$ are coefficients characterizing a momentum transfer from photons to electrons and the degree of electron polarization and the passage from the first into the second layer; $\mu_{B}$ is the Bohr magneton; $\mu$ and $\mu_{0}$ are the magnetic and absolute magnetic permeability; $1_{\mathrm{s}}, 1_{\mathrm{e}}$ are proportionality constant.

At $I=100 \mathrm{MW} / \mathrm{cm}^{2}, \alpha=10^{5} \mathrm{~cm}^{-1}, R=0.5, r=10^{-6}$ $\mathrm{m}, \tau_{\mathrm{s}}=10^{-10}-10^{-11} \mathrm{~s}, \tau_{\mathrm{p}}=10^{-11}-10^{-12} \mathrm{~s}, \mu=10^{4}, \gamma=0.8$, $\eta=0.8, \xi=0.5$ we can obtain the value $H_{\mathrm{s}}>10^{6} \mathrm{~A} / \mathrm{m}$ and $H_{\mathrm{e}}=10^{4}-10^{5} \mathrm{~A} / \mathrm{m}$.

The results of our research have shown that magnetic reversal of the films $\mathrm{Al}_{2} \mathrm{O}_{3} / \mathrm{Tb}_{25} \mathrm{Co}_{5} \mathrm{Fe}_{70} / \mathrm{Al}_{2} \mathrm{O}_{3}$ and $\mathrm{Al}_{2} \mathrm{O}_{3} / \mathrm{Tb}_{19} \mathrm{Co}_{5} \mathrm{Fe}_{76} / \mathrm{Al}_{2} \mathrm{O}_{3}$ with one magnetic nanolayer can be received at an irradiation linearly and circularly polarized picosecond and femtosecond laser pulse without an external magnetic field and in a magnetic field which is directed opposite to initial magnetization of its nanolayer. Characteristic feature of magnetic reversal of these nanolayers under the influence of one circularly polarized femtosecond laser pulse is that magnetic reversal of the nanolayer $\mathrm{Tb}_{19} \mathrm{Co}_{5} \mathrm{Fe}_{76}$ occurs faster than magnetic reversal of the nanolayer $\mathrm{Tb}_{25} \mathrm{Co}_{5} \mathrm{Fe}_{70}$ (Fig. 2).

The observed difference in the reversal of nanolayers $\mathrm{Tb}_{25} \mathrm{Co}_{5} \mathrm{Fe}_{70}$ and $\mathrm{Tb}_{19} \mathrm{Co}_{5} \mathrm{Fe}_{76}$ may be caused by the different contributions of the sublattice magnetization of iron in the total magnetic moment of these nanolayers. In film $\mathrm{Tb}_{19} \mathrm{Co}_{5} \mathrm{Fe}_{76}$, the total magnetic moment is directed toward the sublattice magnetization of iron, and in the film $\mathrm{Tb}_{25} \mathrm{Co}_{5} \mathrm{Fe}_{70}$, it is directed in the direction of magnetization of the terbium sublattice. The reversal magnetic field of the inverse Faraday effect in the nanolayer $\mathrm{Tb}_{19} \mathrm{Co}_{5} \mathrm{Fe}_{76}$ has an opposite direction under the relation of a direction of magnetization of the sublattice of iron in this layer. In the nanolayer $\mathrm{Tb}_{25} \mathrm{Co}_{5} \mathrm{Fe}_{70}$, the similar reversal magnetic field is directed along the direction

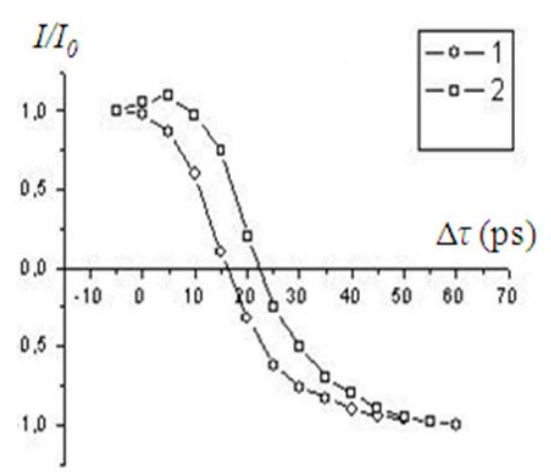

(a)

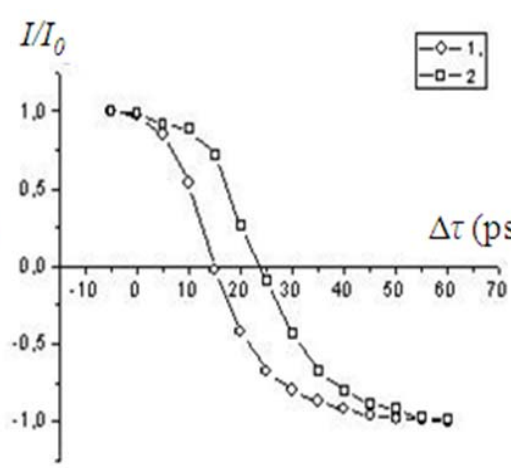

(b)

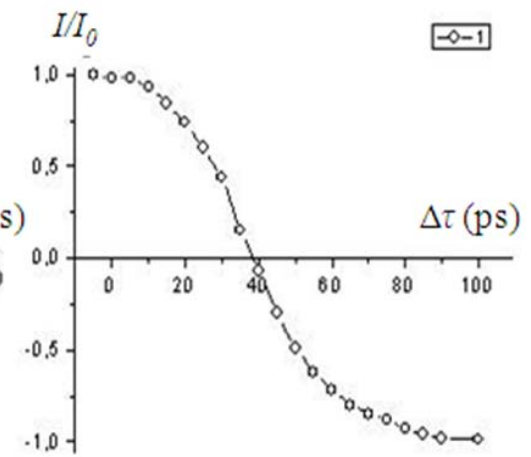

(c)

Fig. 2 The delay time curve of a probe linearly polarized femtosecond pulses transmitted and reflected from a film (a) probe pulse passes through film $\mathrm{Tb}_{19} \mathrm{Co}_{5} \mathrm{Fe}_{76}$ (1) and $\mathrm{Tb}_{25} \mathrm{Co}_{5} \mathrm{Fe}_{70}$ (2), high-power laser pulse irradiates these films is circularly polarized; (b) probe pulse is reflected from $\mathrm{Tb}_{19} \mathrm{Co}_{5} \mathrm{Fe}_{76}$, high-power laser pulse irradiates the film $\mathrm{Tb}_{22} \mathrm{Co}_{5} \mathrm{Fe}_{73} \uparrow$ $/ \mathrm{Pr}_{6} \mathrm{O}_{11} / \mathrm{Tb}_{19} \mathrm{Co}_{5} \mathrm{Fe}_{76} \downarrow$ from $\mathrm{Tb}_{22} \mathrm{Co}_{5} \mathrm{Fe}_{73}$ and has the circular polarization (1) and linear polarization (2); (c) probe pulse is reflected from $\mathrm{Co}_{30} \mathrm{Fe}_{70}$, the high-power laser pulse irradiates the film $\mathrm{Co}_{80} \mathrm{Fe}_{20} \uparrow / \mathrm{Pr}_{6} \mathrm{O}_{11} / \downarrow \mathrm{Co}_{30} \mathrm{Fe}_{70}$ from the $\mathrm{Co}_{80} \mathrm{Fe}_{20}$ and has the circular polarization. 
of magnetization of the sublattice of iron and directed against the direction of magnetization of the terbium sublattice.

As a result, magnetic field of the inverse Faraday effect switch in the nanolayer $\mathrm{Tb}_{19} \mathrm{Co}_{5} \mathrm{Fe}_{76}$ at Firs sub-lattice of iron and in the nanolayer $\mathrm{Tb}_{25} \mathrm{Co}_{5} \mathrm{Fe}_{70}$ sublattice of terbium. And because of the magneto-optical effect in these nanolayers due to the interaction of light with the iron sublattice, we have seen at the time of the circularly polarized femtosecond laser pulse in the nanolayer $\mathrm{Tb}_{25} \mathrm{Co}_{5} \mathrm{Fe}_{70}$ of a slight increase of the Faraday effect in contrast to the nanolayer $\mathrm{Tb}_{19} \mathrm{Co}_{5} \mathrm{Fe}_{76}$.

The magnetic switching of the nanolayers $\mathrm{Tb}_{19} \mathrm{Co}_{5} \mathrm{Fe}_{76}$ and $\mathrm{Tb}_{25} \mathrm{Co}_{5} \mathrm{Fe}_{70}$ (transition via zero the photoelectric signal) occurs faster than $\tau<10$ ps. For the opposite circular polarization or linear polarization of femtosecond laser pulse, the magnetic switching of these nanolayers is not observed without an external magnetic field, even when the intensity of radiation becomes two times bigger.

In the $\mathrm{Tb}_{22} \mathrm{Co}_{5} \mathrm{Fe}_{73} / \mathrm{Pr}_{6} \mathrm{O}_{11} / \mathrm{Tb}_{19} \mathrm{Co}_{5} \mathrm{Fe}_{76}$ film, the magnetization reversal of the first magnetic nanolayer (relative to the irradiating laser pulse) occurs in the same way as in the $\mathrm{Tb}_{22} \mathrm{Co}_{5} \mathrm{Fe}_{73}$. The magnetization reversal of the second magnetic nanolayer depends on the polarization of laser radiation, but also on the state of magnetization in both magnetic layers. In the film with parallel magnetized magnetic nanolayers, $\mathrm{Tb}_{22} \mathrm{Co}_{5} \mathrm{Fe}_{73} \uparrow / \mathrm{Pr}_{6} \mathrm{O}_{11} / \mathrm{Tb}_{19} \mathrm{Co}_{5} \mathrm{Fe}_{76} \uparrow$ can be received the magnetic reversal of the nanolayer $\mathrm{Tb}_{19} \mathrm{Co}_{5} \mathrm{Fe}_{76}$ without the external magnetic field at the irradiation circularly polarized picosecond and femtosecond laser pulse in the result of the inverse Faraday effect.

Temporal characteristics of such magnetic switching are close to dynamic behavior of the magnetic switching in the nanolayer $\mathrm{Tb}_{19} \mathrm{Co}_{5} \mathrm{Fe}_{76}$ differing only in a power-level of the laser radiation. In such film with antiparallel magnetized nanolayers, $\mathrm{Tb}_{22} \mathrm{Co}_{5} \mathrm{Fe}_{73} \uparrow / \operatorname{Pr}_{6} \mathrm{O}_{11} / \mathrm{Tb}_{19} \mathrm{Co}_{5} \mathrm{Fe}_{76} \downarrow$ can be received the magnetic reversal of the output reversal nanolayer
$\mathrm{Tb}_{19} \mathrm{Co}_{5} \mathrm{Fe}_{76}$ without the external magnetic field at the irradiation not only for the circular or for linear polarization picosecond and femtosecond laser pulses (Fig. 2b), but it is reached only when the laser pulse falls on a film from the layer $\mathrm{Tb}_{22} \mathrm{Co}_{5} \mathrm{Fe}_{73}$.

At the radiation of the film $\mathrm{Tb}_{22} \mathrm{Co}_{5} \mathrm{Fe}_{73} \uparrow / \mathrm{Pr}_{6} \mathrm{O}_{11} / \mathrm{Tb}_{19} \mathrm{Co}_{5} \mathrm{Fe}_{76} \downarrow$ from the side of the nanolayer $\mathrm{Tb}_{19} \mathrm{Co}_{5} \mathrm{Fe}_{76}$ linearly polarized picosecond and femtosecond pulses, we could not get a reversal of the output nanolayer $\mathrm{Tb}_{22} \mathrm{Co}_{5} \mathrm{Fe}_{73}$ without external magnetic field.

The results of the investigations of the films $\mathrm{Tb}_{22} \mathrm{Co}_{5} \mathrm{Fe}_{73} \uparrow / \mathrm{Pr}_{6} \mathrm{O}_{11} / \mathrm{Tb}_{19} \mathrm{Co}_{5} \mathrm{Fe}_{76} \downarrow$ with antiparallel nanolayer magnetizations show that the magnetic reversal of the layer $\mathrm{Tb}_{19} \mathrm{Co}_{5} \mathrm{Fe}_{76}$ linearly polarized laser pulses is due to the magnetic field $H_{i}=H_{e}+H_{s}$ of the spin polarized electrons injected into the layer from the nanolayer $\mathrm{Tb}_{22} \mathrm{Co}_{5} \mathrm{Fe}_{73}$ by laser radiation. Our films are characterized by a large perpendicular anisotropy. Therefore, the effect of the magnetic field $H_{e}$ on magnetic switching of the nanolayers is minimal.

At the irradiation of the films $\mathrm{Co}_{30} \mathrm{Fe}_{70} / \mathrm{Al}_{2} \mathrm{O}_{3}$ with one ferromagneticnanolayer with the small angular dispersion $\left(\Delta \alpha \approx 3^{\circ}\right)$ of planar single-axis magnetic anisotropy, the magnetic switching of nanolayer takes place only in an external magnetic field, which is directed opposite to the magnetization of the magnetic nanolayer $\mathrm{Co}_{30} \mathrm{Fe}_{70}$. In the film $\mathrm{Co}_{80} \mathrm{Fe}_{20} \uparrow / \mathrm{Pr}_{6} \mathrm{O}_{11} / \downarrow \mathrm{Co}_{30} \mathrm{Fe}_{70} \quad$ with antiparallel magnetizations of the nanolayers, it is possible to get the magnetic switching of nanolayer $\mathrm{Co}_{30} \mathrm{Fe}_{70}$ without magnetic field. Such magnetic switching of nanolayer $\mathrm{Co}_{30} \mathrm{Fe}_{70}$ can be received only at the film $\mathrm{Co}_{80} \mathrm{Fe}_{20} \uparrow / \mathrm{Pr}_{6} \mathrm{O}_{11} / \downarrow \mathrm{Co}_{30} \mathrm{Fe}_{70}$ irradiation picosecond and femtosecond laser pulses from the nanolayer $\mathrm{Co}_{80} \mathrm{Fe}_{20}$. For very high radiation intensity of the circularly or linearly polarized femtosecond laser pulses the delay time curve for the reflected linearly polarized probe laser pulse passes through zero and becomes negative (Fig. 2c). The magnetic switching of the low-coercive nanolayer $\mathrm{Co}_{30} \mathrm{Fe}_{70}$ without the 
external magnetic field takes place under the action of the internal effective magnetic field $H_{i}=H_{e}+H_{s}$ laser-induced spin current caused by a photon drag effect of laser pulse. The magnetic nanolayers $\mathrm{Co}_{80} \mathrm{Fe}_{20}$ and $\mathrm{Co}_{30} \mathrm{Fe}_{70}$ have a planar single-axis magnetic anisotropy, therefore the self-field $H_{e}$ of the electric current and the magnetic field $H_{s}$ related to the total magnetic moment of the injection spins have the direction in the plane of nanolayer $\mathrm{Co}_{30} \mathrm{Fe}_{70}$. In this case, the value of the internal effective magnetic field is the sum of these two fields $H_{\mathrm{i}}=\mathrm{k}_{\mathrm{s}} H_{s}+\mathrm{k}_{\mathrm{e}} H_{e}$, where, $\mathrm{k}_{\mathrm{s}}$ and $\mathrm{k}_{\mathrm{e}}$ are constants. The magnetic switching of the nanolayer $\mathrm{Co}_{30} \mathrm{Fe}_{70}$ occurs in a time of about $\tau=20 \mathrm{ps}$.

The research of influencing of femtosecond laser pulse on resistance of microcontacts $\mathrm{Tb}_{19} \mathrm{Co}_{5} \mathrm{Fe}_{76} / \mathrm{Pr}_{6} \mathrm{O}_{11} / \mathrm{Tb}_{22} \mathrm{Co}_{5} \mathrm{Fe}_{73} \quad$ and $\mathrm{Co}_{80} \mathrm{Fe}_{20} / \mathrm{Pr}_{6} \mathrm{O}_{11} / \mathrm{Co}_{30} \mathrm{Fe}_{70}$ at the absence of magnetic field showed that at small intensity of laser radiation, the resistance of microcontacts falls at the moment of action of laser pulse, but after finishing of the pulse, it returns back to the practically initial value.

At a large intensity of laser radiation, the resistance of these microcontacts changes after the end of the laser pulse and this change depends on the state of magnetization of magnetic layers, on the intensity, and even on the incidence direction of laser pulses on the contact. As our research showed, the magnetic reversal of one of the electrodes changes the relative resistance change in the microcontacts $\mathrm{Co}_{80} \mathrm{Fe}_{20} / \mathrm{Pr}_{6} \mathrm{O}_{11} / \mathrm{Co}_{30} \mathrm{Fe}_{70}$ values $\Delta R / R>0.15$ at $T=300 \mathrm{~K}$ and $\Delta R / R>0.5$ at $T=$ $80 \mathrm{~K}$. In the microcontacts $\mathrm{Tb}_{22} \mathrm{Co}_{5} \mathrm{Fe}_{73} / \mathrm{Pr}_{6} \mathrm{O}_{11}$ $/ \mathrm{Tb}_{19} \mathrm{Co}_{5} \mathrm{Fe}_{76}, \Delta R / R>0.5$ at $T=300 \mathrm{~K}$ and $\Delta R / R>1.4$ at $T=80 \mathrm{~K}$.

In the microcontacts $\mathrm{Co}_{80} \mathrm{Fe}_{20} \uparrow / \mathrm{Pr}_{6} \mathrm{O}_{11} / \uparrow \mathrm{Co}_{30} \mathrm{Fe}_{70}$ with parallel magnetizations of the nanolayers, it is impossible to get the change of resistance after radiation of a laser pulse in the absence of the magnetic field. In the microcontact $\mathrm{Co}_{80} \mathrm{Fe}_{20} \uparrow / \operatorname{Pr}_{6} \mathrm{O}_{11} / \downarrow \mathrm{Co}_{30} \mathrm{Fe}_{70}$ with antiparallel magnetizations of the nanolayers, the change of resistance at a radiation of the picosecond and femtosecond laser pulses occurs (Fig. 3).

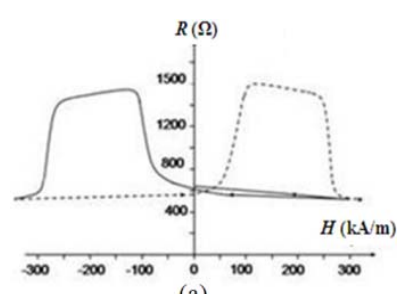

(a)

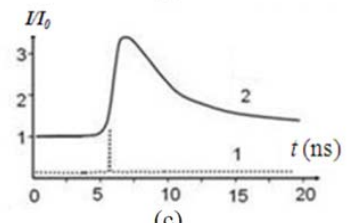

(c)

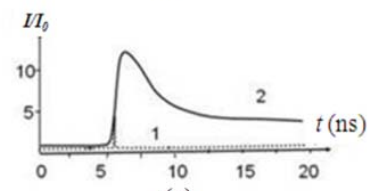

(e)

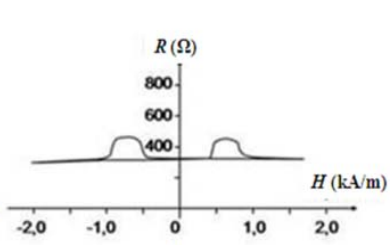

(b)

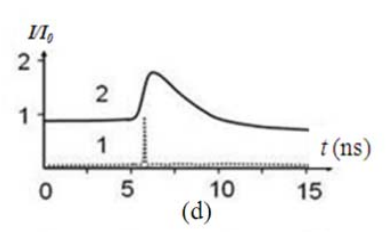

(d)

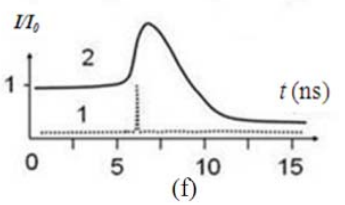

Fig. 3 The change of resistance of tunnel contact $\mathrm{Tb}_{22} \mathrm{Co}_{5} \mathrm{Fe}_{73} / \mathrm{Pr}_{6} \mathrm{O}_{11} / \mathrm{Tb}_{19} \mathrm{Co}_{5} \mathrm{Fe}_{76} \quad$ (a, c, e) and $\mathrm{Co}_{80} \mathrm{Fe}_{20} \uparrow / \mathrm{Pr}_{6} \mathrm{O}_{11} / \downarrow \mathrm{Co}_{30} \mathrm{Fe}_{70}$ (b, d, f) at their magnetic switching of an external magnetic field $(a, b)$ and under the influence of circularly polarized femtosecond laser pulse (c-f) at $T=300 \mathrm{~K}(\mathrm{a}-\mathrm{d})$ and $T=80 \mathrm{~K}(\mathrm{e}, \mathrm{f})$.

Such change of resistance in microcontact $\mathrm{Co}_{80} \mathrm{Fe}_{20} \uparrow / \mathrm{Pr}_{6} \mathrm{O}_{11} / \downarrow \mathrm{Co}_{30} \mathrm{Fe}_{70}$ can be received only at an irradiation of this microcontact from the side of the nanolayer $\mathrm{Co}_{80} \mathrm{Fe}_{20}$. The laser pulses directed from resistance do not take place. In the microcontacts, of $\mathrm{Tb}_{22} \mathrm{Co}_{5} \mathrm{Fe}_{73} / \mathrm{Pr}_{6} \mathrm{O}_{11} / \mathrm{Tb}_{19} \mathrm{Co}_{5} \mathrm{Fe}_{76}$ at an irradiation circularly polarized picosecond and femtosecond laser pulses, it is possible to receive change of conductivity of contact without an external magnetic field both at parallel and at antiparallel magnetization of the magnetic nanolayers.

The laser-induced change conductivity of the microcontacts $\mathrm{Tb}_{22} \mathrm{Co}_{5} \mathrm{Fe}_{73} \uparrow / \operatorname{Pr}_{6} \mathrm{O}_{11} / \uparrow \mathrm{Tb}_{19} \mathrm{Co}_{5} \mathrm{Fe}_{76}$ is related to magnetic switching of nanolayer $\mathrm{Tb}_{19} \mathrm{Co}_{5} \mathrm{Fe}_{76}$ in the magnetic field of the inverse Faraday. The laser-induced change conductivity of the microcontacts $\mathrm{Tb}_{22} \mathrm{Co}_{5} \mathrm{Fe}_{73} \uparrow / \operatorname{Pr}_{6} \mathrm{O}_{11} / \downarrow \mathrm{Tb}_{19} \mathrm{Co}_{5} \mathrm{Fe}_{76}$ is related to magnetic switching of nanolayer $\mathrm{Tb}_{19} \mathrm{Co}_{5} \mathrm{Fe}_{76}$ in the magnetic field of the inverse Faraday effect and in the magnetic field $H_{s}$ related to the total magnetic moment of the injection in this layer polarized electrons. 
The difference in intensities of radiation of the circular polarization picosecond laser pulses inducing a change conductivity of contacts $\mathrm{Tb}_{22} \mathrm{Co}_{5} \mathrm{Fe}_{73} / \mathrm{Pr}_{6} \mathrm{O}_{11} / \mathrm{Tb}_{19} \mathrm{Co}_{5} \mathrm{Fe}_{76}$ is explained by the fact that magnetic switching of the input magnetic nanolayer $\mathrm{Tb}_{19} \mathrm{Co}_{5} \mathrm{Fe}_{76}$ takes place only under the influence of the magnetic field of inverse Faraday effect $H_{F}$, while the magnetic switching of the output magnetic nanolayer is caused the sum of this field $H_{F}$ and field $H_{s}$ related to the total magnetic moment of the injection spins.

The results of investigation show that the laser-induced switching can be realized not only for the case of perpendicular but also uniaxial planar magnetic anisotropy of the magnetic nanolayers. This fact allows us to use the mechanism of magnetization reversal of the control electrodes in spintronic elements and to write data to the spin storage medium.

Based on these results, we have developed and produced experimental models of the devices for recording data on a spin storage media [16]. The scheme of such storage spin media and the storage cell of this storage media are shown in Fig. 4. The storage cell of this media has two tunneling microcontacts (Fig. 4a): (1) first electrode from magnetic material; (2) barrier nanolayer from isolating material; (3) second electrode from magnetic material and (4) third electrode from magnetic material.

The electrodes of these contacts should be made of

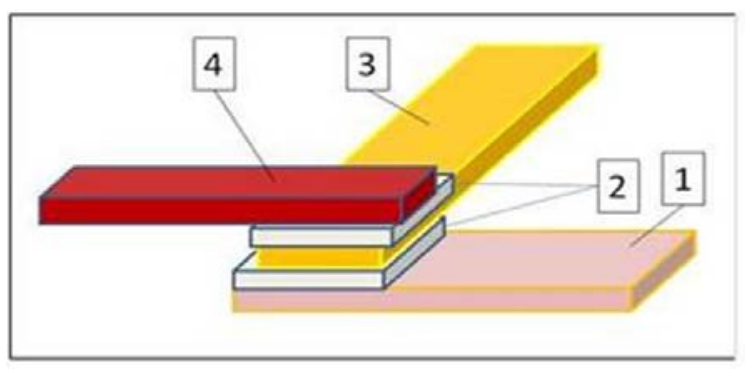

(a)

Fig. 4 (a) The scheme of storage cell; (b) storage spin media. 0 is substrate, 1 is first magnetic electrode, 2 is insulating nanolayer, 3 is the second magnetic stripe electrode, 4 is the third magnetic stripe electrode. magnetic material with a high degree of spin polarization of electrons and it should have perpendicular anisotropy or uniaxial planar magnetic anisotropy in the plane. Coercive force $H_{1}$ of the first electrode is bigger than coercive force $\mathrm{H}_{2}$ of the second electrode, and coercive force $H_{3}$ of the third electrode is bigger than coercive force of the first electrode: $H_{1}>$ (1.2-1.4) $H_{2}$ and $H_{3}>(1.2-1.3) H_{1}$. Different values of coercive force make it possible to magnetize magnetic electrodes 1 and 3 of our storage cell in the opposite direction by means of external magnetic field.

For the data recording of our cell, high-power electric nanosecond pulse energizes. The data recording in our storage cell in the form of " 0 " or " 1 " was spent by means of nanosecond electric pulses. For the recording of the information as " 1 " nanosecond electric pulses to the first and second magnetic electrodes our cell must be supplied. For putting down the information in the form of " 0 " nanosecond electrical pulse to the third and second magnetic electrodes our cell must be supplied. In both cases, a positive pulse must be supplied to the second electrode.

As the material for barrier nanolayer, it is better to use $\mathrm{MgO}$ or $\operatorname{Pr}_{6} \mathrm{O}_{11}$. The magnesium oxide has a special electronic structure that provides record high values of tunnel magnetoresistance $(T M R>300 \%)$ in the tunnel magnetic contacts [17]. The praseodymium oxide is a high energy-gap semiconductor which at the

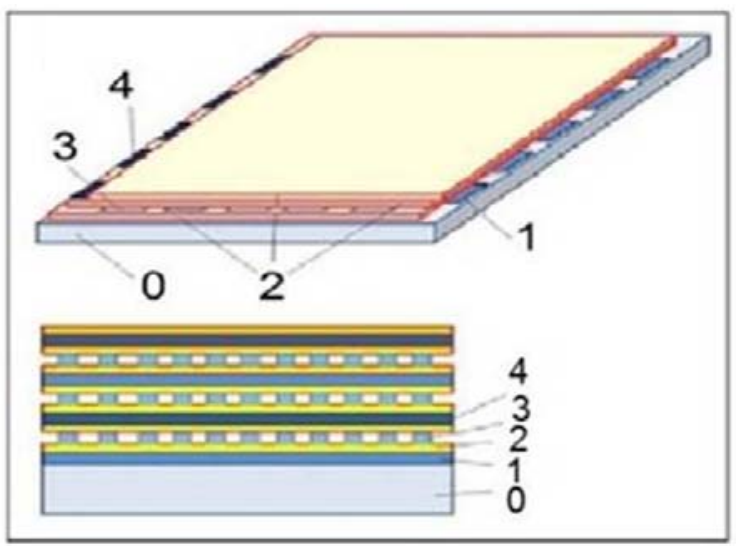

(b) 
room temperature is in the paramagnetic state. If we place a thin nanolayer $\operatorname{Pr}_{6} \mathrm{O}_{11}$ between two ferromagnetic layers, the nanolayer $\operatorname{Pr}_{6} \mathrm{O}_{11}$, it is magnetized at the expense of penetration of polarized electrons into it [18].

Under the influence of this electric field, the pulse of spin current is excited in the tunnel junction. The polarized electrons are injected from the electrode 1 or from the electrode 3 to the electrode 2 and create nonequilibrium magnetic field which magnetizes this electrode in the direction of magnetization of the first or third electrode. Pulse amplitude of the current which should pass through the contact 1-2 or 3-2 can be obtained from Eq. (2):

$$
J_{s}>\frac{H_{a} 4 \pi \mu_{0} S_{c} h e}{\gamma \tau_{s} \mu_{B} \mu}, J_{e}>2 \pi \frac{\mu_{0}}{\mu} H_{a} \sqrt{S_{c}}
$$

where, $J_{s}$ and $J_{e}$ amplitude of spin current and electric current, $S_{c}$, contact area; $h$, thickness of nanolayer 2; $e$ and $\tau_{s}$, electron charge and spin polarization relaxation time in electrode $2 ; \mu$ and $\mu_{0}$, magnetic permeability of electrode 3 and the absolute magnetic permeability; $\gamma<1$, factor which characterizes degree of spin polarization in electrode 1 or 4. Estimates show that at $J_{s}=10^{-6} \mathrm{~A}$ in contact with $S_{c}=1 \mu \mathrm{m}^{2}, h=40 \mathrm{~nm}, \tau_{s}=10^{-9} \mathrm{~s}, \mu=500 \mathrm{H} / \mathrm{m}$ and $\gamma=$ 0.5 spin current generates high magnetic field $H_{s}$ is around $10^{7} \mathrm{~A} / \mathrm{m}$ and $H_{e}>10^{6} \mathrm{~A} / \mathrm{m}$ which is much higher than most field anisotropy of magnetic films.

Differential method of reading the information is used in our cell. The differential amplifier registers the electric signal $U_{s}$ as equal to the difference of conductivity of the tunnel contact 1-2 and the tunnel contact 3-2 $U_{s}=k\left(J_{32}-J_{12}\right)$. At the recording, a single electric nanosecond pulse is applied simultaneously to the electrodes 1 and 3. The electrode 2 is used as a common electrode. The amplitude of the read pulse must be much smaller than the amplitude of the write pulse. Conductivity of our tunneling contact depends on the relative orientation of the magnetization vectors in the electrodes that contact [19]:

$$
J=e^{2} h^{-1}|T|^{2}=A e^{2} h^{-1}\left(1+P^{2} \cos \theta\right) \exp (-2 \kappa s)
$$

where, $e$ is the electron charge, $\theta$ is angle between the magnetization vectors of the magnetic electrodes in this contact

$$
P=\left[N_{\uparrow}(E)-N_{\downarrow}(E)\right] /\left[N_{\uparrow}(E)+N_{\downarrow}(E)\right], \kappa=\sqrt{2 m^{*}\left(U-E_{F}\right) / \hbar^{2}}
$$

is the state density in the ferromagnetic at the Fermi level $\left(E_{F}\right)$ for spin up and spin down; $s, m^{*}$ and $U$ are the barrier thickness, effective electron mass respectively, and $A$ is a proportionality coefficient.

It follows that, when " 1 " is written down " 1 " in our storage cell, we receive positive value $U_{s}$ ("1") $>0$ of the electric signal after the differential amplifier and when " 0 " is written down in the memory cell, we receive a negative value $U_{s}$ (" 0 ") $<0$ of the signal of reading after the differential amplifier.

On the basis of such storage cell, it is possible to make a multilayered spin carrier of the information (Fig. 4b). Such spin carrier of the information consists of a system of $\mathrm{klm}$ storage cell. The very small volume of the storage cell in such spin carrier gives a chance to reach a very high information capacity with the small size of this carrier. In the described spin carrier of information, it is possible to make the information record-reading by parallel way that considerably increases the speed of such process.

We have made an experimental sample of the storage cell. In this cell, ferromagnetic nanolayers with perpendicular anisotropy $\mathrm{Tb}_{22} \mathrm{Co}_{5} \mathrm{Fe}_{73} / \mathrm{Pr}_{6} \mathrm{O}_{11} / \mathrm{Tb}_{17} \mathrm{Co}_{5} \mathrm{Fe}_{78} / \mathrm{Pr}_{6} \mathrm{O}_{11} / \mathrm{Tb}_{19} \mathrm{Co}_{5} \mathrm{Fe}_{76}$ were used. Experimental samples were prepared as following. On the substrate platinum electrodes, nanolayer $\mathrm{Tb}_{22} \mathrm{Co}_{5} \mathrm{Fe}_{73}$ thickness of $40-45 \mathrm{~nm}$ and nanolayer $\operatorname{Pr}_{6} \mathrm{O}_{11}$ thickness about $1 \mathrm{~nm}$ were deposited. Then by laser lithography, the electrodes $\mathrm{Tb}_{22} \mathrm{Co}_{5} \mathrm{Fe}_{73}$ with the width of $2 \mu \mathrm{m}$ were formed. On these electrodes, nanolayer $\operatorname{Pr}_{6} \mathrm{O}_{11}$ thickness of $1 \mathrm{~nm}$ and nanolayer $\mathrm{Tb}_{17} \mathrm{Co}_{5} \mathrm{Fe}_{78}$ thickness of $30-35 \mathrm{~nm}$ then again deposited nanolayer $\operatorname{Pr}_{6} \mathrm{O}_{11}$ thickness of $1 \mathrm{~nm}$ were deposited. Then by laser lithography formed the 
electrodes $\mathrm{Tb}_{17} \mathrm{Co}_{5} \mathrm{Fe}_{78}$ with the width of $2 \mu \mathrm{m}$, which were oriented perpendicular to the first electrodes $\mathrm{Tb}_{22} \mathrm{Co}_{5} \mathrm{Fe}_{73}$. These electrodes was deposited nanolayer $\operatorname{Pr}_{6} \mathrm{O}_{11}$ thickness of $1 \mathrm{~nm}$, and nanolayer $\mathrm{Tb}_{19} \mathrm{Co}_{5} \mathrm{Fe}_{76}$ of thickness 40-45 $\mathrm{nm}$ then again deposited nanolayer $\operatorname{Pr}_{6} \mathrm{O}_{11} 1 \mathrm{~nm}$. Then by laser lithography formed the electrodes $\mathrm{Tb}_{19} \mathrm{Co}_{5} \mathrm{Fe}_{76}$ width of $2 \mu \mathrm{m}$ that were a directed parallel to the electrodes $\mathrm{Tb}_{22} \mathrm{Co}_{5} \mathrm{Fe}_{73}$ first magnetic nanolayer and placed exactly opposite to these electrodes. On these layers, deposited protective layer $\operatorname{Pr}_{6} \mathrm{O}_{11}$ thickness of $20 \mathrm{~nm}$.

\section{Conclusions}

The analysis of the features of laser interaction with thin films outlines perfect prospects for using laser radiation for high-speed spin current control in spintronic elements. Our experimental studies of laser interaction with multilayer magnetic nanofilms have shown that magnetic reversal of magnetic nanolayers under the action of picosecod and femtosecond laser pulses occurs in time near $\tau$ (around $10^{-11} \mathrm{~s}$ ) very fast. The analysis of the results also shows that there are various physical mechanisms of magnetic reversal of magnetic films under the influence of laser radiation. The basic ones are the following: thermomagnetic switching under the influence of an external magnetic field or a demagnetization field, magnetic switching of antiferromagnetic films under the influence of an effective internal field of antiferromagnetic interaction between magnetic sublattices rare-earth and transitive metals, magnetic switching under the influence of a magnetic field of the inverse Faraday effect, or under the influence of a magnetic field on a spin current. In multilayer magnetic films, the photon drag effect leads to the spin current in the direction of laser radiation. The laser-induced injection of the spin-polarized electrons can cause non-equilibrium magnetization in the nonmagnetic semiconductor and metal layers. It may also lead to a reversal of the magnetic nanolayers.

The magnetic switching of magnetic layers under action of the magnetic field of a spin current is extremely important for practical use in elements of spintronics. This mechanism of magnetic reversal takes place only in multilayer nanofilms. The great advantage of the magnetization reversal of magnetic nanolayers of the spin current is that the mechanism of magnetization reversal works in the films with perpendicular anisotropy and in the films with in-plane anisotropy.

The injection of polarized electrons can also be realized by using short electrical pulses. That is why the mechanism of magnetization reversal of the control electrodes spin current is the most appropriate for the management of high-spin current in the elements of spintronics. On the basis of this mechanism that has been performed by us, a high-speed recording of the information on the spin carrier and the experimental model memory with three magnetic nanolayers was obtained. The data recording in this memory cell is realized by using an electric nanosecond pulse. This recording can be realized by using picosecond electrical pulses.

\section{References}

[1] Zuti'c, Fabian, J., and Sarma, S. D. 2004. "Spintronics: Fundamentals and Applications." Rev. Mod. Phys. 76 (2): 323-410.

[2] Cibert, J., Bobo, J., and Lüders, U. 2005. "Development of New Materials for Spintronics." Comptes. Rendus. Physique 6: 977-96.

[3] Leamy, H. J., and Dirks, A. G. 1979. "Microstructure and Magnetism in Amorphous Rare-Earth-Transition-Metal Thin Films, II Magnetic Anisotropy.” J. Appl. Phys. 50 (4): 2871-82.

[4] Krupa, M. M., Kuzmak, O. M., and Karaseva, V. Y. 2001. "Characteristics of Formation Perpendicular Magnetic Anisotropy in Films of Alloys Rare-Earth and Transitive Metals." Surface (Russia) 11: 92-7.

[5] Krupa, M. M. 2009. "Spin Dependent Current and Magnetization Reversal in $\mathrm{Tb}_{22} \mathrm{Co}_{5} \mathrm{Fe}_{73} / \mathrm{Pr}_{6} \mathrm{O}_{11}$ $/ \mathrm{Tb}_{19} \mathrm{Co}_{5} \mathrm{Fe}_{76}$ Nanofilms in Laser Radiation Field." JETPh (Russia) 108 (5): 856-65.

[6] Chen, P., Moser, J., Kotissek, P., Sadowski, J., Zenger, M., Weiss, D., and Wegscheider, W. 2006. "All-Electrical Measurement of Spin Injection in a Magnetic p-n Junction Diode." Phys. Rev. B 74 (24): 241-302. 
[7] Krupa, M. M. 2012. "Switching of Magnetic Films by Femtosecond Laser Pulses and Control Spin Current." Advances in Optoelectronic Materials 1 (3): 48-58.

[8] Stanciu, C. D., Kimel, A. V., Hansteen, F., Tsukamoto, A., Itoh, A., Kirilyuk, A., and Rasing, T. 2006. "Ultrafast Spin Dynamics across Compensation Points in Ferromagnetic GdFeCo: The Role of Angular Momentum Compensation." Phys. Rev. B 73 (22): 220402 (1)-(4).

[9] Stanciu, C. D., Kimel, A. V., Hansteen, F., Tsukamoto, A., Itoh, A., Kirilyuk, A., and Rasing, T. 2009. "Inertiadriven Spin Switching in Antiferromagnets." Nature Physics 5: 727-31.

[10] Pershan, P. S., Ziel, J. P., and Malmstrom, L. D. 1996. "Theoretical Discussion of the Inverse Faraday Effect, Raman Scattering, and Related Phenomena." Phys. Rev. 143 (2): 574-83.

[11] Hertel, R. 2006. "Theory of Optical Rotation, Faraday Effect and Inverse Faraday Effect.” Journal of Magnetism and Magnetic Materials 303: L1-L4.

[12] Slonczewski, J. C. 1996. "Current-Driven Excitation of Magnetic Multilayers." Journal of Magnetism and Magnetic Materials 159: L1-L7.
[13] Katine, J., Albert, F., Buhrman, R., Myers, E. B., and Ralph, D. C. 2000. "Current-Driven Magnetization Reversal and Spin-Wave Excitations in $\mathrm{Co} / \mathrm{Cu} / \mathrm{Co}$ Pillar." Phys. Rev. Letters 84: 3149-52.

[14] Askaryan, G. A., Rabiovych, M. C., Smirnova, A. D., and Studentov, V. B. 1967. "The Currents Generated in the Material by Radiation Pressure of the Laser Beam." Sov. Phys. JETFh Letters 5: 116-8.

[15] Krupa, M. M. 2001. "Light Induced Drift Electrons in Thin Magnetic Films.” JETPh (Russia) 120 (11): 10-5.

[16] Krupa, M. M. 2014. A Method of Magnetic Recording Media and Magnetic Spin Media. Ukraine Patent 109,260, field August, 2012 and issued August 112014.

[17] Yuasa, S., and Djayaprawira, D. D. 2007. "Giant Tunnel Magnetoresistance in Magnetic Tunnel Junctions with a Crystalline $\mathrm{MgO}(001)$ Barrier." J. Phys. D: Appl. Phys. 40: R337.

[18] Krupa, M. M. 2008. "Spin-Dependent Tunneling Conductance in $\mathrm{TbCoFe} / \mathrm{Pr}_{6} \mathrm{O}_{11} / \mathrm{TbCoFe}$ Films." JETP Letters 87 (10): 608-12.

[19] Julliere, M. 1975. "Tunneling between Ferromagnetic Films.” Phys. Letter. A 54: 225. 